\title{
Fuzzy-Based Sensor Fusion for Cognitive Radio-Based Vehicular Ad Hoc and Sensor Networks
}

\author{
Mohammad Jalil Piran, Amjad Ali, and Doug Young Suh \\ Electronics and Radio Engineering Department, Kyung Hee University, 1732 Deogyeong-daero, Giheung-gu, Yongin-si, \\ Gyeonggi-do 446-701, Republic of Korea \\ Correspondence should be addressed to Doug Young Suh; suh@khu.ac.kr
}

Received 3 November 2014; Accepted 3 February 2015

Academic Editor: Chunlin Chen

Copyright (C) 2015 Mohammad Jalil Piran et al. This is an open access article distributed under the Creative Commons Attribution License, which permits unrestricted use, distribution, and reproduction in any medium, provided the original work is properly cited.

In wireless sensor networks, sensor fusion is employed to integrate the acquired data from diverse sensors to provide a unified interpretation. The best and most salient advantage of sensor fusion is to obtain high-level information in both statistical and definitive aspects, which cannot be attained by a single sensor. In this paper, we propose a novel sensor fusion technique based on fuzzy theory for our earlier proposed Cognitive Radio-based Vehicular Ad Hoc and Sensor Networks (CR-VASNET). In the proposed technique, we considered four input sensor readings (antecedents) and one output (consequent). The employed mobile nodes in CR-VASNET are supposed to be equipped with diverse sensors, which cater to our antecedent variables, for example, The Jerk, Collision Intensity, and Temperature and Inclination Degree. Crash_Severity is considered as the consequent variable. The processing and fusion of the diverse sensory signals are carried out by fuzzy logic scenario. Accuracy and reliability of the proposed protocol, demonstrated by the simulation results, introduce it as an applicable system to be employed to reduce the causalities rate of the vehicles' crashes.

\section{Introduction}

Wireless sensor networks (WSNs) are composed of a large number of sensor nodes, which are constrained in power and communication range and are having multimodal sensing capacity. The tiny motes consist of sensing, data processing, and communication components, which leverage on the idea of sensor networks based on the collaborative effort of a large number of nodes [1]. The operations of the network are performed under environmental conditions, which are characterized by low signal-to-noise ratio, interference, and multipath effects [2]. The nodes are randomly distributed over a region to detect a physical phenomenon or an event. The harsh fields and power constraint may make some sensor nodes inoperable. Hence, the network's lifetime mainly depends on the power source. Due to the network constraints, it is necessary to find out some techniques that improve the flow of information. One of the suggested solutions is data aggregation or data fusion. Data fusion techniques provide a single data by collecting a set of various source data. Data fusion can reduce the amount of data flowing and the energy consumed for data processing and transmission by eliminating redundant data. As a definition, data fusion is a process of combining information from several sources to reduce beneficiary and reliable information. In WSNs, data fusion can be achieved by deletion of redundancy, energy consumption, and assuring fault-tolerance among sensor nodes.

In order to reduce nodes' power consumption and consequently enlarge the network life time, numerous research works considered data aggregation and sensor fusion techniques, such as those regarding aggregation, metadata negotiation, or data fusion [3-8]. The authors in [3] address the fusion problem in WSNs where the cross-correlation between the estimates is unknown. With the assumptions that the covariance matrix has a prior distribution and also information about the covariance of each estimate is known, the conditional distribution of the offdiagonal blocks is derived. In [4], the authors focused on a data aggregation mechanism to the number of transmissions and thereby minimize energy consumption. For congestion 
control, a fusion-centric scheme is proposed in [7]. While most of the other approaches focused on controlling link level congestions, the authors in [7] proposed a scheme to control the congestion at node level. A hybrid energy-efficient data aggregation protocol for large scale WSNs is proposed in [8], which uses the spatial and temporal correlation of the data for effective aggregation and thereby preserving energy. The proposed scheme in [5] performs filtration of sensed data by eliminating the redundancy in the sensed data pattern of the nodes using Brownian motion. In [6], information fusion is used to improve the accuracy of location estimation in WSNs with less computation complexity.

We already, in [9], have introduced a new system called cognitive radio-based vehicular ad hoc and sensor networks (CR-VASNET). In our system there are two kinds of sensor nodes, some are embedded on the vehicles (called as mobile nodes) and others are deployed at a predetermined distance beside the highway road, known as road side sensor (RSS), whereas the generated packet by the sensor nodes is time sensitive and must be received in a minimum time by the base station (BS), and because of spectrum scarcity issue, CR-VASNET nodes are equipped with cognitive radio technology. Cognitive radio enables the nodes to utilize licensed spectrum bands in an opportunistic manner with any harmful interference to the incumbents. These mobile nodes are used to sense the vehicle's activity. There are some BSs, such as Police Traffic station, Firefighting Group, or Rescue Team. The BSs may be stationary or mobile. We have already considered diverse aspects of the proposed CRVASNET such as routing, localization, and spectrum access, in [9]. In this paper we focus on sensor fusion aspect to detect vehicles crashes.

Whereas fuzzy logic methods have the ability of fusing uncertain data from several sources to improve the quality of information, we employed fuzzy inference model for our sensor fusion protocol, in order to estimate crash (accident) severity in CR-VASNET. The mobile nodes attached to the vehicles provide required antecedent variables, that is, the jerk, collision intensity, temperature, and inclination degree. And the system provides the crash severity as consequent variable. The generated consequent as an accident alarm is then transmitted to the BSs. Based on the crash severity the required type of rescue will be decided by rescue team (BSs). This paper presents the research further undertaken to fuse information from the mobile nodes to make composite information from them more robust and make the overall crash detection system more reliable.

The rest of the paper is organized as follows. Section 2 describes our system framework. Simulation and performance evaluation are discussed in Section 3. And finally, Section 4 concludes the paper.

\section{System Framework}

When an accident occurs especially on the highway roads, informing the relevant authorities, for example, rescue team, firefighting group, and traffic police within minimum time, is an important issue to save injured people. In CR-VASNET, sensor nodes are employed to detect and notify vehicles' crashes. When the nodes detect collision or overturns, immediately a packet will be generated and transmitted towards the BSs. For example, if a simple collision takes place with no damages, then there is no need for the attention of the firefighting, rescue team, or ambulance. Hence, the details about the accident (severity) such as what kind and level of accident have taken place should also be considered and conveyed for relevant action which in turn saves time and resources.

In general, fuzzy logic is a multivalue logic, by which intermediate values can be defined using expressions, such as true/false, high/low, and below/above [10]. As shown in Figure 1, data fusion process is completed in four phases: (1) fuzzification, (2) rule evaluation, (3) combination and aggregation of rules, and (4) defuzzification. In our system, the jerk, collision intensity, inclination degree, and also temperature are the input fuzzy variables (antecedents) and the crash (accident) severity is the output variable (consequent).

As abstracted in Figure 1, we have to devise an interpolation mechanism that is capable of generating a numeric output from a numeric set of inputs after considering all the rules in the rule base. Our fuzzy control system comprises the following modules. (1) Fuzzification interface: it fuzzifies the crisp inputs by assigning grades of membership using fuzzy sets defined for that particular variable. (2) Fuzzy rule base: it consists of a "database" and a "linguistic control rule base." The database is maintained to provide the required definitions to define linguistic control rules and fuzzy data manipulation. It characterizes the control goals and control strategies of the domain experts by means of a set of linguistic control rules. (3) Fuzzy inference engine: it infers output by employing the fuzzy implications and the rule of inference of fuzzy logic. And (4) defuzzification, which yields a nonfuzzy control action from an inferred control action. We use (IF antecedent THEN consequent) function for our system.

Table 1, shows some examples, where $J, T$ and $C$ are the input values (jerk, temperature, and collision, resp.) and $j 1$, $j 2, j 3, c 2$ and $t 1$ are the terms for input variables, and CS (Crash Severity) is output variable with values $a 1, a 2$, and $a 3$. There are three famous and common fuzzy logic inference methods, for example, Mamdani, Tsukamoto, Sugeno and Larsen, which work with crisp data as input [11]. These methods are different in terms of fuzzy rules consequents, aggregations, and defuzzification procedures. Mamdani method has been proposed to control a steam engine and boiler using a set of linguistic control rules. In Mamdani method, inputs are crisp values and by using defuzzification, a fuzzy set will be converted to crisp value. In the Tsukamoto method, the output of each fuzzy rule is represented using a fuzzy set with a monotonic feature while Sugeno method was proposed to develop a systematic technique to produce fuzzy rules from a given input or output dataset. In this paper we follow the most commonly used Mamdani method [12] instructions, because it allows us to describe the expertise in more intuitive and more human-like manner.

As the first step in the Mamdani method, we assign a degree of membership for each input value to the appropriate defined fuzzy set. The membership functions LOW, MEDUIM, HIGH, and VERY HIGH are defined on each input variable and VERY LOW, LOW, MEDUIM, HIGH, 
TABLE 1: Some examples of system's rules.

\begin{tabular}{lc}
\hline Rule & Rule description using crisp values \\
\hline 1 & IF $J$ is $j_{1}$ OR $C$ is $c_{2}$, THEN CS is $a_{1}$ \\
2 & IF $J$ is $j_{2}$ AND $T$ is $t_{1}$, THEN CS is $a_{2}$ \\
3 & IF $J$ is $j_{3}$, THEN CS is $a_{3}$ \\
\hline
\end{tabular}

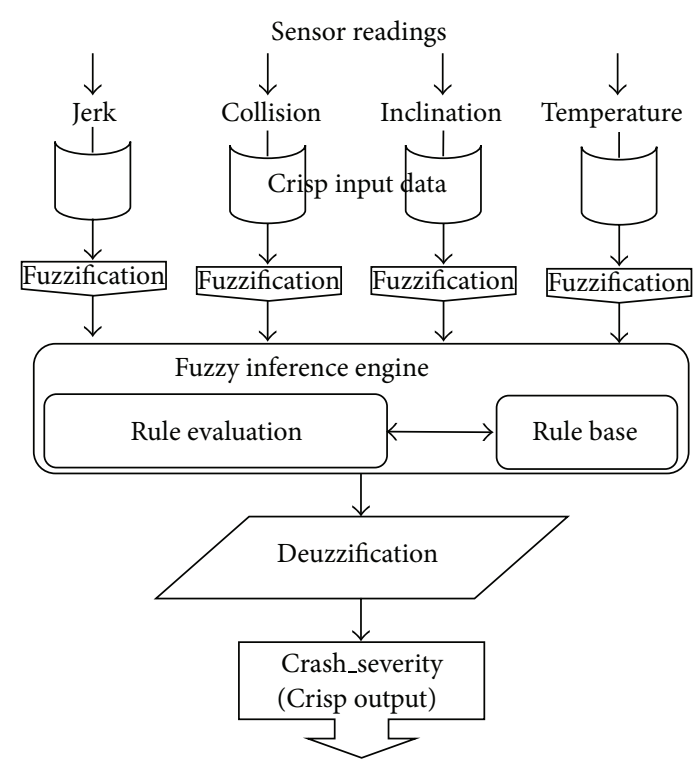

Figure 1: Flow of data in our fuzzy control system.

and VERY HIGH are defined on output variables. There are several types of membership function in fuzzy, for example, triangular, trapezoidal, Gaussian, Bell, Sigmoidal, Asymmetric, and L-R. We used triangular, trapezoidal, and Gaussian for our model. The advantages of using these three member functions are as follows: (1) defining of the membership functions based on a small amount of data, (2) simplicity in parameters modification based on antecedents' measured values to obtain the consequent, (3) the possibility of obtaining input and output mapping of the model, a hypersurface composed on linear segments, and (4) polygonal membership functions meaning the condition of a partition of unity whereas the sum of membership grades for each value must be one, by using polygonal membership functions it can be easily achieved. As mentioned already, we have four input variables as our antecedents: (1) jerk, (2) collision intensity, (3) inclination degree, and (4) temperature. In the following subsections, we explain how to obtain the crisp value of each antecedent as well as fuzzification assignment for each one.

2.1. Jerk. The velocity is defined as speed of movement of an object:

$$
v=\frac{\Delta \bar{x}}{\Delta t},
$$

where " $v$ " is velocity, " $x$ " is position, and " $t$ " stands for time. The acceleration, $a$, is the differentiation of velocity with respect to the time:

$$
\vec{a}=\frac{\Delta \bar{v}}{\Delta t} \quad \text { or } \quad \vec{a}=\frac{d \vec{v}}{d t}=\frac{d^{2} \vec{x}}{d t^{2}} .
$$

The jerk or derivative of acceleration is the first order differentiation of acceleration with respect to time, or the second derivative of velocity, or the third derivative of the position. The jerk is directly related to a physical damage process of materials and structures [13]. We measure the instantaneous acceleration, for example, by accelerometer, and then, by taking the difference with respect to the time, compute the time rate of change of acceleration, which is the jerk. Meter per second cubed is the unit of jerk $\left(\mathrm{m} / \mathrm{s}^{3}\right)$ :

$$
\overrightarrow{\jmath_{t}}=\frac{d \bar{a}_{(t)}}{d t}=\vec{a}_{(t)}=\frac{d^{2} \vec{v}_{(t)}}{d t^{2}}=\ddot{\vec{v}}_{(t)}=\frac{d^{3} \vec{x}_{(t)}}{d t^{3}}=\ddot{\vec{r}}_{(t)} .
$$

According to [14], the jerk in 3D motion consists of three components that can be expressed as

$$
\begin{aligned}
\frac{d a}{d t}= & \frac{d^{3} x}{d t^{3} T}+\frac{d\left(k V^{2}\right)}{d t N} \\
& +k\left|V^{3}\right|\left(-k T+\left(\frac{1}{|V|^{2}}\right)\left(\frac{d|V|}{d t}\right) N+\Upsilon B\right),
\end{aligned}
$$

where $T$ is the unit vector tangent to the curve, pointing in the direction of motion, $N$ is the normal unit vector, $B$ is the binormal unit vector and the cross product of $T$ and $N$, and $k$ is used to effectively define the curvature and torsion of a space curve. The first expression is the rate of change of linear acceleration, the second expression is normal acceleration change rate, and the last term is the fundamental Frenet vector. $\Upsilon$ is the only parameter that cannot be determined without $d a / d t$. Generally, the jerk's conventional measurement works as follows: a signal input, which can be a displacement or a force excitation, is detected or measured by an accelerometer. The numerical differentiation of the measured acceleration yields the jerk output [15]. The output of the jerk sensor is a voltage proportional to jerk, which can be measured by a jerk sensor. When an accident happens, two cars collide, the situation is more complex, and both vehicles produce negative acceleration and jerk. Negative acceleration makes passengers jerk in the forward direction and at times even cause casualty and death. Jerk is the cause of parts' molecular rupture, resulting in destruction. The fuzzy input jerk is fuzzified using triangular and trapezoidal functions as shown in Figure 2, where the LOW, MEDIUM, HIGH, and VERY HIGH components represent magnitude of participation for the input. Trapezoidal and triangular membership functions have been chosen because they have been shown to be a good compromise between effectiveness 


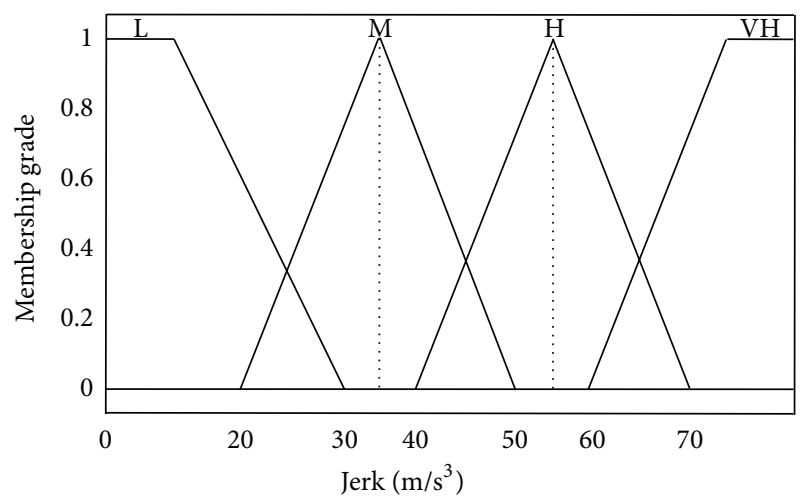

Figure 2: Fuzzification assignment for the jerk input variable.

and efficiency. These, trapezoidal and triangular membership functions can be calculated as follows:

$$
\begin{gathered}
\text { tringle }(x ; a, b, c)=\max \left(\min \left(\frac{x-a}{b-a}, \frac{c-x}{c-b c}\right), 0\right), \\
\text { trapezoid }(x ; a, b, c, d)=\max \left(\min \left(\frac{x-a}{b-a}, 1, \frac{d-x}{d-c}\right), 0\right),
\end{gathered}
$$

where $a_{i}, b_{i}, c_{i}$, and $d_{i}$ are coordinates of the $i$ th trapezoid apexes for fuzzy set $A$.

2.2. Collision Intensity. Collisions with a static obstacle or a mobile vehicle are two cases when a crash happens. We measure the intensity of the collision with respect to the mass of the vehicle and also its velocity, which is equal to the kinetic energy:

$$
k=\frac{1}{2} m v^{2},
$$

where " $m$ " is the mass of the vehicle and " $v$ " is the velocity. The metric of the collision is Joules. The fuzzy input collision intensity is fuzzified using triangular and trapezoidal functions as shown in Figure 3.

2.3. Inclination Degree. Inclination degree or the angle of the vehicle with respect to gravity can be found based on the following formula:

$$
\alpha=\arcsin \left(\frac{g_{x}}{-g}\right),
$$

where " $g_{x}$ " is the acceleration due to gravity in the " $x$ " direction.

Table 2 represents mapping for a universe of discourse of inclination of the vehicle to degrees of the possibility that a vehicle is overturned. Figure 4 shows the fuzzy set defined on the universe of discourse, inclination degree using Gaussian function.

2.4. Temperature. As shown in Figure 5, the fuzzy sets for temperature input variable are defined by triangular and

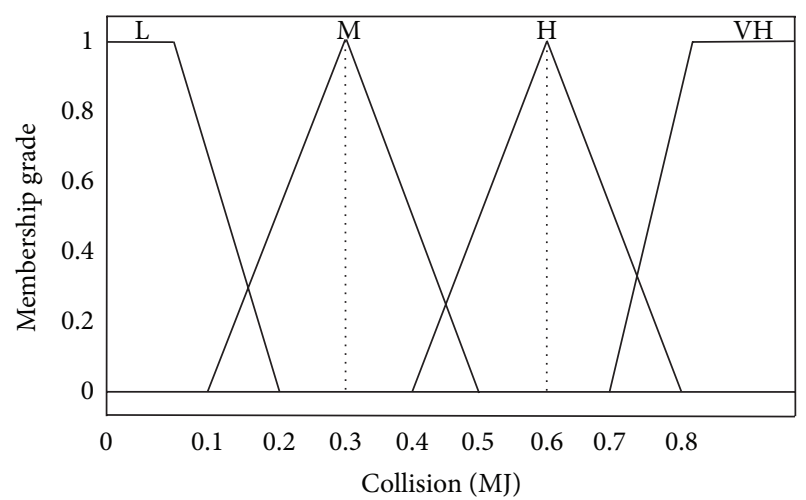

FIgURE 3: Fuzzification assignment for the collision intensity input variable.

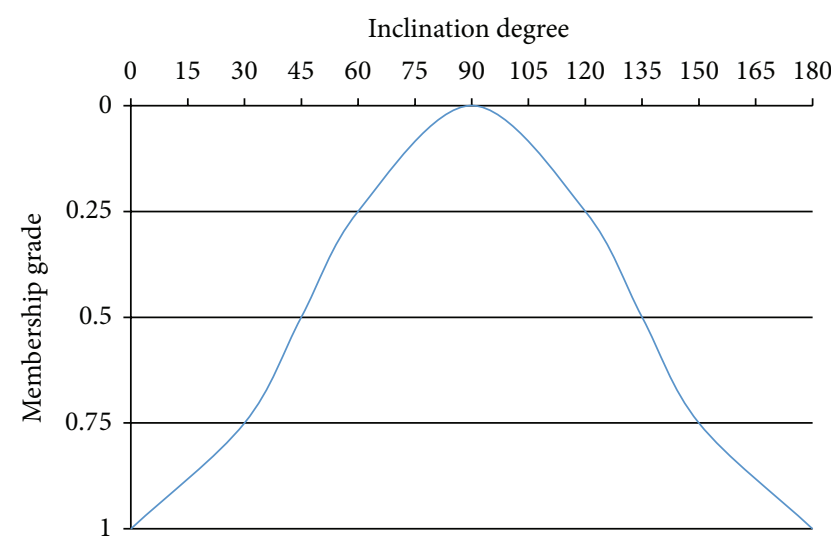

FIgURE 4: Fuzzification assignment for the inclination degree input variable.

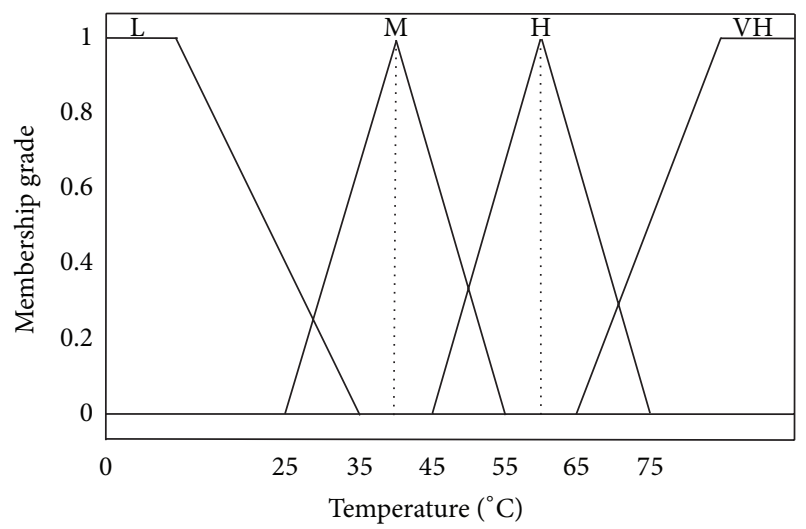

FIGURE 5: Fuzzification assignment for the temperature input variable.

trapezoidal functions. The core of the set defined as the most representative element of that set is defined as core $A=\{x \mid$ $\left.x \in X, \mu_{A}=1\right\}$. Table 3 shows the core of our fuzzy sets.

The four input variables are used to assign a weight factor to each sensor that detects an event. The input fuzzy values are saved using array vectors. By the formed array vectors, we try to eliminate redundancy data coming from 

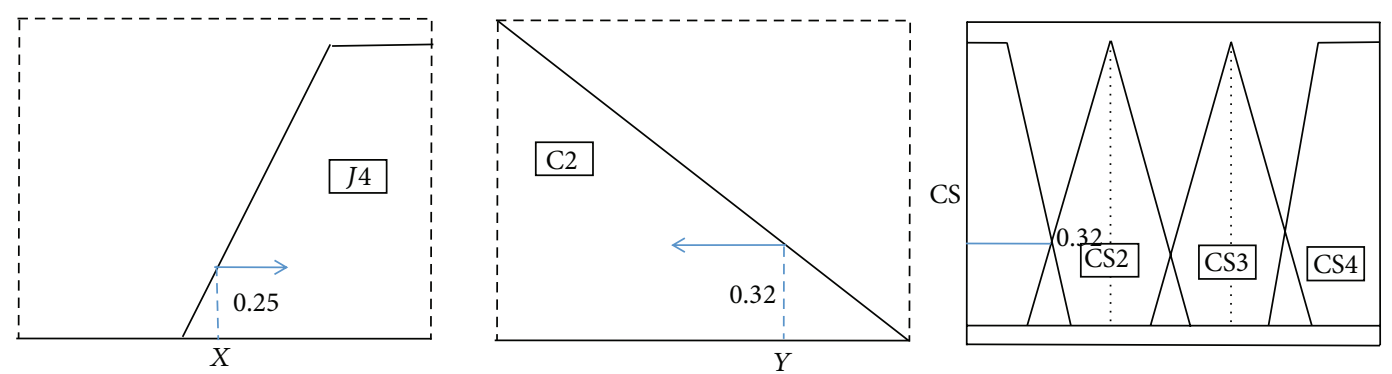

(a) IF X IS J4 (0.25) OR (MAX) Y IS C2 (0.32) THEN CS IS 0.32
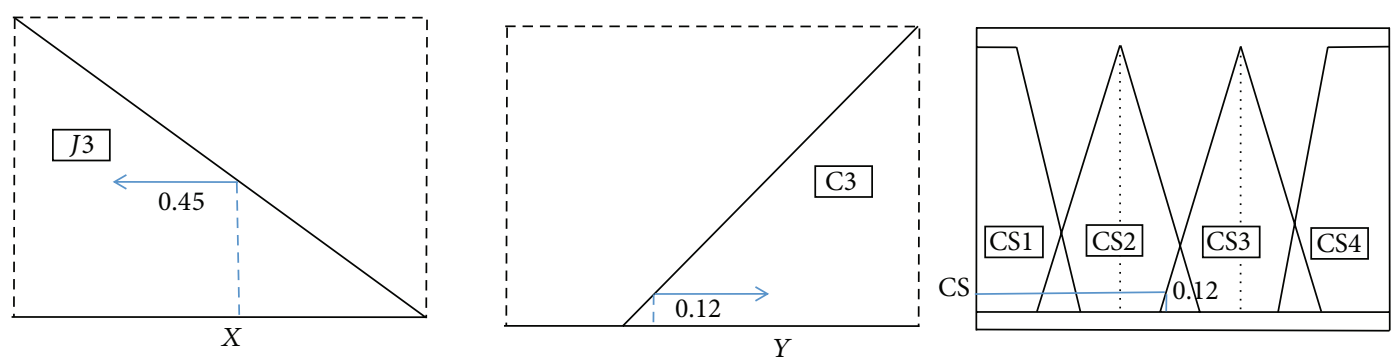

(b) IF X J3 (0.45) AND (MIN) Y IS C3 (0.12) THEN CS IS 0.12

FiguRE 6: Rule evaluation process.

TABLE 2: The possibility that a vehicle is overturned.

\begin{tabular}{lc}
\hline Inclination (angle) & Mem. Fun. degree \\
\hline $180^{\circ}$ & 1 \\
$150^{\circ}$ & 0.75 \\
$135^{\circ}$ & 0.5 \\
$120^{\circ}$ & 0.25 \\
$90^{\circ}$ & 0 \\
$60^{\circ}$ & 0.25 \\
$45^{\circ}$ & 0.5 \\
$30^{\circ}$ & 0.75 \\
$0^{\circ}$ & 1 \\
\hline
\end{tabular}

TABLE 3: Core of our fuzzy sets.

\begin{tabular}{lccc}
\hline Variable/range & $J$ & $C$ & $T$ \\
\hline $\mathrm{L}$ & $<15$ & $<0.07$ & $<10$ \\
$\mathrm{M}$ & 35 & 0.3 & 40 \\
$\mathrm{H}$ & 55 & 0.6 & 60 \\
$\mathrm{VH}$ & $>75$ & $>0.8$ & $>85$ \\
\hline
\end{tabular}

the same sensors. Then, the second step is the rule evaluation, where the fuzzified inputs are applied according to certain appropriate rules. The logic operators, for example, (AND) and (OR), which represent minimum (intersection) and maximum (union), respectively, are used to estimate a crisp value. This value describes the result after the rule evaluation, in case where a fuzzy rule has more than one condition element, as depicted by Figures 6(a) and 6(b). The numerical values, such as minimum, maximum, mean, and standard deviation of the input fuzzy magnitudes, are used to select the center and width used in triangular, trapezoidal, and Gaussian functions during the second step.

As an example we draw the graph in case $J$ and $C$ are 63.3 and 0.73 , respectively. Based on triangular and trapezoidal membership function assignment: $\mu_{H} 63.3=0.45, \mu_{\mathrm{VH}} 63.3=$ 0.25 , and, $4 ; \mu_{M} 0.73=0.32$ and $\mu_{H} 0.73=0.12$.

The aggregation of all outputs is the third step. Combination of the outputs of each rule to compose a new fuzzy set is the most considerable issue in this step. See Figure 7.

As we are going to estimate the severity of crash (accident), Figure 8 describes the expected fuzzy output variable (crash severity), where $\mathrm{VL}, L, M, H$, and $\mathrm{VH}$ represent the magnitude of participation for that output.

The conversion of the aggregated new fuzzy set into a number (crisp value) is the final step, which is known as the defuzzification. The mentioned conversion can be done by the Centroid technique. As shown in Figure 9, determination of the point, at which a vertical line split the combined set into two parts, is known as the Centroid technique. The Centroid method is given by the following formula [11]:

$$
Z=\frac{\int \mu_{A}^{\prime}(z) * z d z}{\int \mu_{A}(z) d z}
$$

where $\mu_{A}(z)$ is the membership function of set $A$. Consider the following:

$$
\begin{aligned}
\mathrm{COG}= & (((0+10+20+30+40) \times 0.32) \\
& +((40+50+60+70+80) \times 0.12)) \\
& \cdot(0.32 \times 5+0.12 \times 5)^{-1} \\
= & 28.72 .
\end{aligned}
$$




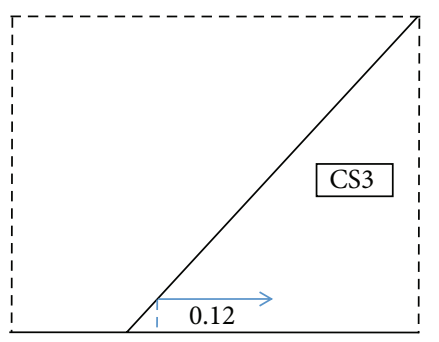

CS is 0.12

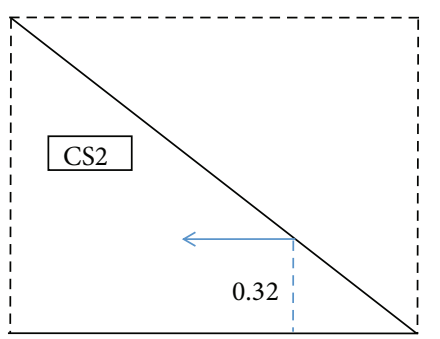

CS is 0.32

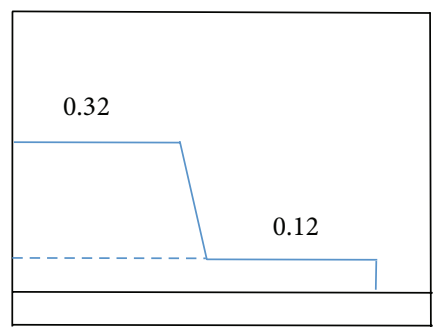

Figure 7: Aggregation of rule outputs.

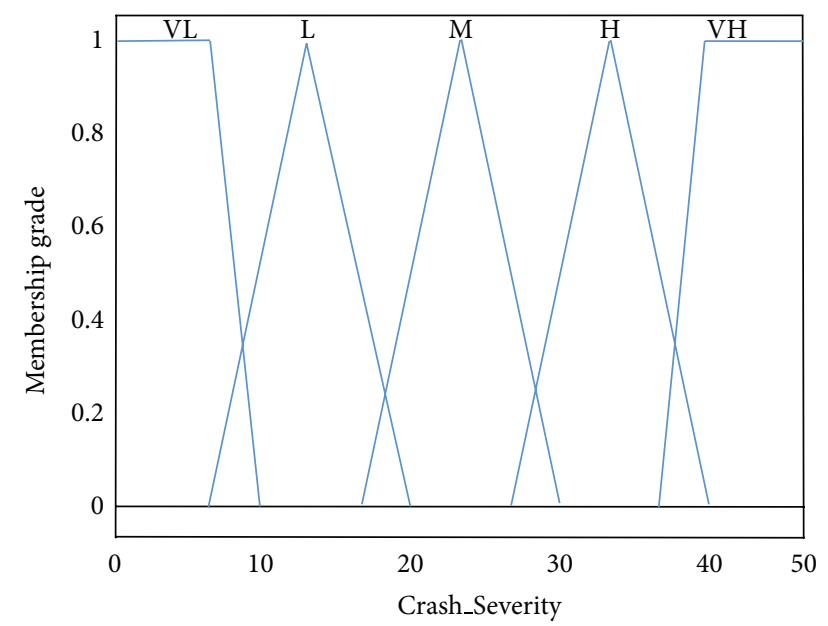

FIGURE 8: Membership function for crash severity.

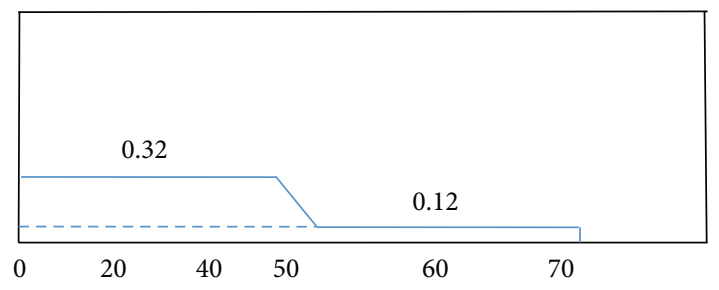

FIGURE 9: Defuzzification process.

\section{Simulation and Results}

The fuzzy (IF antecedent THEN consequent) rules in expert systems are defined as follows:

IF $J$ is $j_{1}$ and $\mathrm{C}$ is $c_{1}$, THEN CS is $\mathrm{cs}_{1}$, else

IF $J$ is $j_{2}$ and $C$ is $c_{2}$ OR $T$ is $t_{1}$, THEN CS is $\mathrm{cs}_{2}$,

where $J, C$, and $T$ are the antecedent variables and CS is a single consequent variable. There are four input variables and each consists of four linguistic variables. Therefore, the total $4^{4}=256$ rules are used, which are all possible combinations of antecedents. Some of the example rules in the rule based system are given below:

(i) IF (jerk is $L$ ) and (collision is $L$ ) and (temperature is $L$ ) and (inclination is not high), THEN (Crash_Severity is $L$ ),

(ii) IF (jerk is $L$ ) and (collision is $L$ ) and (temperature is M) OR (inclination is high), THEN (Crash_Severity is $M)$,

(iii) IF (jerk is $M$ ) OR (collision is $L$ ) and (temperature is $L$ ) and (inclination is high), THEN (Crash_Severity is $H)$,

(iv) IF (jerk is $H$ ) OR (collision is $H$ ) and (temperature is $\mathrm{VH}$ ) and (inclination is high), THEN (Crash_Severity is $\mathrm{VH})$.

The rules are created using the fuzzy inference system (FIS) editor contained in the MATLAB Fuzzy Toolbox. Figure 10 depicts a sample fuzzy calculation of crash severity based on the amount of the jerk, collision intensity, temperature, and inclination degree. Variations of member function of each input variable are shown in this figure as well. The red line indicates the assumed value for the variable.

In our system, diverse sensors detect event parameters by setting threshold values. To save the power, the sensed data by diverse sensors is compared to the threshold value, and only if it is decided to transmit the data, it will trigger the transmitter and will transmit to the BS via RSS. The processor part of the sensor receives the parameters (jerk, collision intensity, inclination degree, and temperature) by these diverse sensors and will compute the mean value of the parameters. Based on the mean value of the sensor-sensed data, the fuzzy logic system generates a numerical value as the crash severity. Table 4 shows a sample output of crash severity. From the last row of the table, it can be seen that jerk is high (65), collision intensity is high (0.18), temperature is very high (90), inclination degree is (0), and then crash severity is very high.

As stated already, the consequent, Crash_Severity, is calculated based on the four antecedents. We have shown how to calculate the consequent based on all antecedents, for example, the case illustrated by Figure 10. However, because of limitation for preparing more than 3-dimensional figures, the following figures depict Crash_Severity calculation based on any two antecedents. Figure 11 shows consequent 

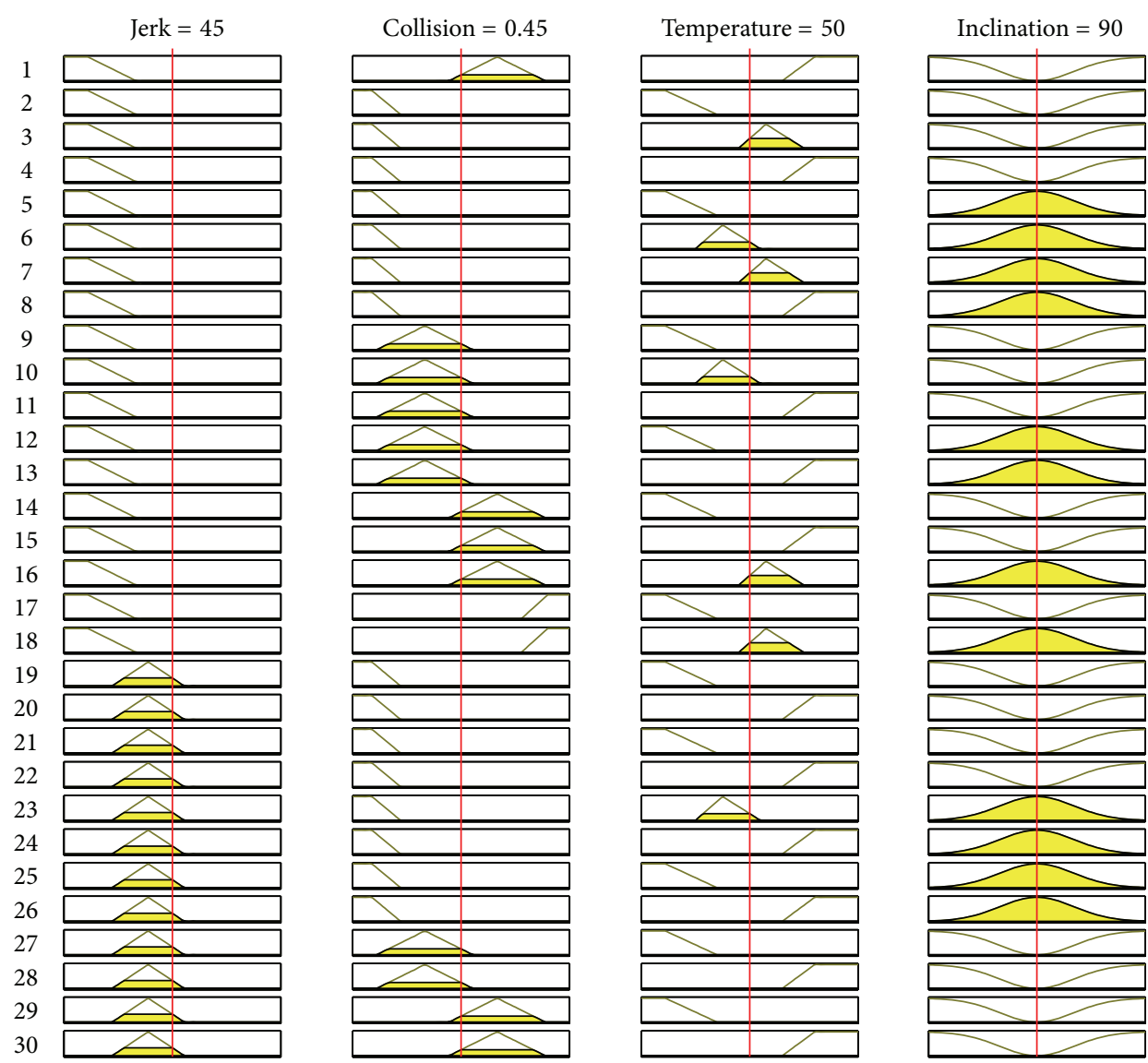

Crash_Severity $=25$

FIGURE 10: Membership function and sample fuzzy calculation for crash severity.

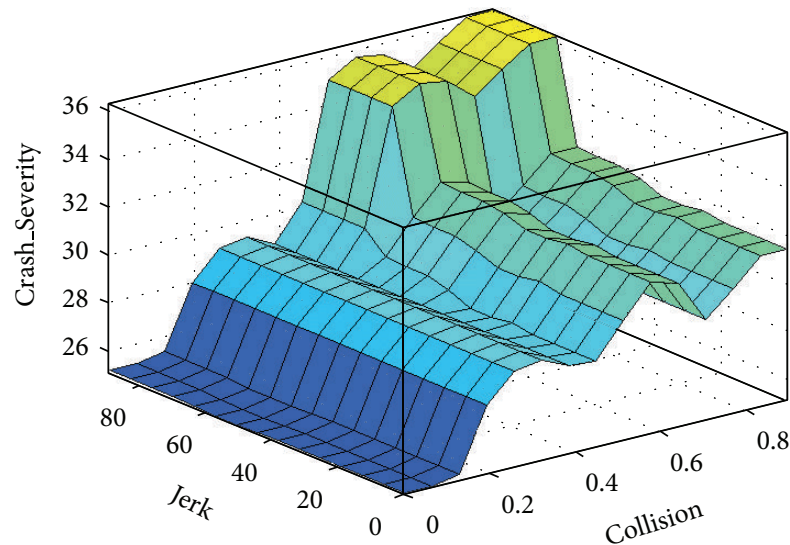

FIGURE 11: Surface view of crash severity with respect to collision intensity and the jerk.

calculation with respect to collision intensity and the jerk. When the two input values are low, the Crash_Severity is also low. Whereas increasing of the jerk and collision intensity is coherent, it means increasing of the jerk results in increasing of collision intensity, and then Crash_Severity will increase too. As shown in Figure 12, only occurrence of jerk when it is less than $70 \mathrm{~m} / \mathrm{s}^{3}$ does not cause severe accident. However,

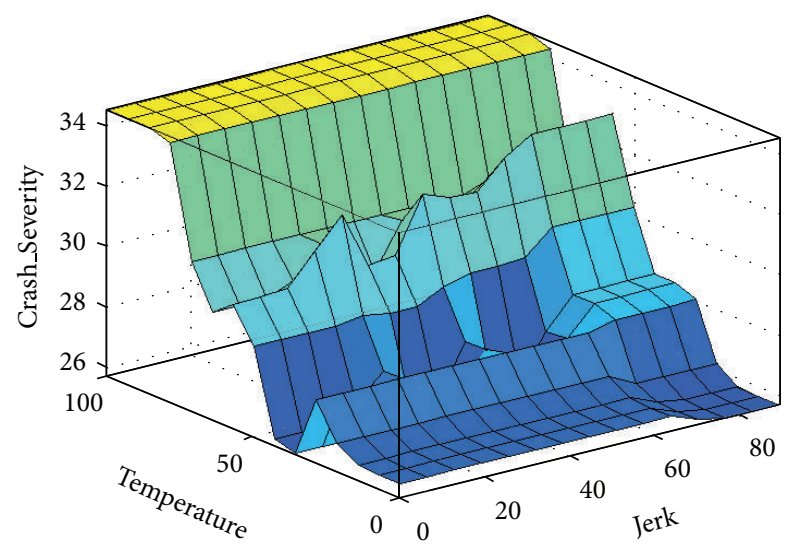

FIGURE 12: Surface view of crash severity with respect to the jerk and temperature.

if only temperature is high or simultaneously both the jerk and temperature increase, the Crash_Severity increases as well. In this case, whenever temperature sensor reports high temperature, it means fire takes place and thereby an alarm message will be sent to firefighting group to go to the accident area. 
TABLE 4: Some output samples of crash severity.

\begin{tabular}{lccccc}
\hline Number & Jerk & Collision intensity & Temperature & Inclination degree & Crash severity \\
\hline 1 & 8 & 0.07 & 12 & 90 & 15 \\
2 & 12 & 0.05 & 37 & 85 & 25 \\
3 & 32 & 0.13 & 10 & 50 & 35 \\
4 & 65 & 0.18 & 90 & 0 & 48 \\
\hline
\end{tabular}

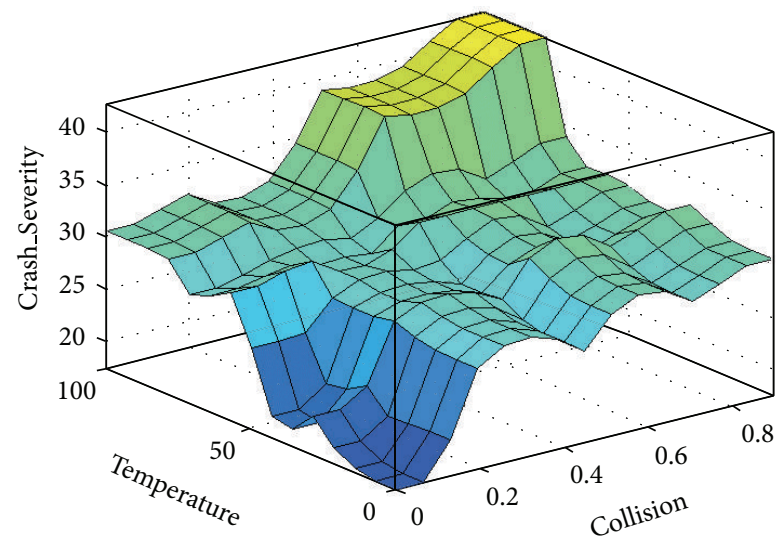

FIGURE 13: Surface view of crash severity with respect to collision intensity and temperature.

Figure 13 shows surface view of Crash_Severity with respect to collision intensity and temperature. Severity of the crash increases when temperature exceeds 50 centigrade. However, with respect to the collision intensity, even when the temperature is less than 50 centigrade, and the collision intensity is high, there may be an accident that occurred. And if both of them increase, Crash_Severity increases as well.

Figure 14 illustrates dependency of Crash_Severity on inclination degree and temperature. As it can be seen, when inclination degree of the vehicle is close to 90 degrees and temperature is less than 50 centigrades, the severity is zero or less, but when the inclination degree tends to 0 or 180 degree and/or temperature increases, then Crash_Severity increases as well. The graph well illustrates the cases where the type of the accident is vehicle overturn only and there is no fire (inclination degree close to 0 or 180 degrees but temperature is low, resulting in increasing the crash severity).

\section{Conclusion}

In this paper we proposed a system for crash severity detection by a fuzzy-based sensor fusion technique for our earlier proposed cognitive radio-based vehicular ad hoc and sensor networks (CR-VASNET). We assumed four sensor readings as the antecedents, that is, the jerk, collision intensity, temperature and inclination degree, and crash severity as the consequent variable. The proposed system effectively handles the uncertainty and vagueness present in the vehicular environment data. The system improves the accuracy of crash detection and severity measurement by processing multiple sensor readings. According to the simulation results it can

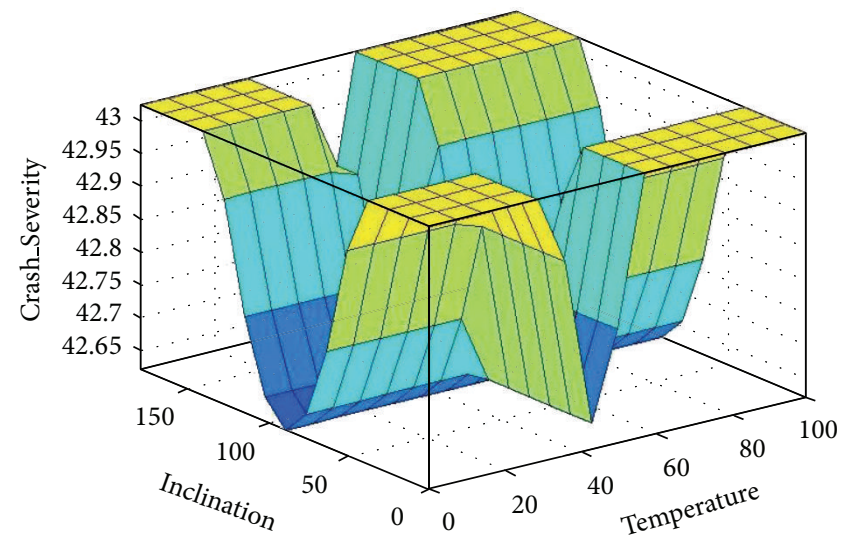

FIGURE 14: Surface view of crash severity with respect to temperature and inclination degree.

be concluded that employing the proposed scheme helps to provide more accurate data to the authorities in comparison to using data from independent sensors.

\section{Conflict of Interests}

The authors declare that there is no conflict of interests regarding the publication of this paper.

\section{Acknowledgment}

This research was funded by the MSIP (Ministry of Science, ICT and Future Planning), Republic of Korea, in the ICT R\&D Program 2015.

\section{References}

[1] I. F. Akyildiz, W. Su, Y. Sankarasubramaniam, and E. Cayirci, "A survey on sensor networks," IEEE Communications Magazine, vol. 40, no. 8, pp. 102-114, 2002.

[2] A. Sinha and A. Chandrakasan, "Dynamic power management in wireless sensor networks," IEEE Design and Test of Computers, vol. 18, no. 2, pp. 62-74, 2001.

[3] Z. Weng and P. Djuric, "A bayesian approach to data fusion in sensor networks," http://arxiv.org/abs/1303.2414. Its eprint eprint arXiv:1303.2414, http://adsabs.harvard.edu//abs/2013arXiv1303.2414W.

[4] C.-M. Chao and T.-Y. Hsiao, "Design of structure-free and energy-balanced data aggregation in wireless sensor networks," Journal of Network and Computer Applications, vol. 37, no. 1, pp. 229-239, 2014. 
[5] A. Sinha and D. K. Lobiyal, "A multi-level strategy for energy efficient data aggregation in wireless sensor networks," Wireless Personal Communications, vol. 72, no. 2, pp. 1513-1531, 2013.

[6] L. Tang, Y. Gong, Y. Luo, S. Feng, and X. Zhao, "Positioning algorithms by information fusion in wireless sensor networks," Wireless Personal Communications, vol. 74, no. 2, pp. 545-557, 2014.

[7] N. Prabakaran, K. Naresh, and R. J. Kannan, "Fusion centric decision making for node level congestion in wireless sensor networks," in ICT and Critical Infrastructure: Proceedings of the 48th Annual Convention of Computer Society of India, vol. 1, Springer International Publishing, 2014.

[8] S. Santhosh and K. Paulose Jacob, "HEAP: hybrid energyefficient aggregation protocol for large scale wireless sensor networks," International Journal Of Computers \& Technology, vol. 4, no. 2, pp. 713-721, 2013.

[9] M. Jalil Piran, Y. W. Cho, J. H. Yun, A. Ali, and D. Y. Suh, "Cognitive radio-based vehicular Ad Hoc and sensor networks," International Journal of Distributed Sensor Networks, vol. 2014, Article ID 154193, 11 pages, 2014.

[10] G.-J. Klir and B. Yuan, Fuzzy Sets and Fuzzy Logic: Theory and Applications, Prentice Hall, New Jersey, NJ, USA, 1995.

[11] T. J. Ross, Fuzzy Logic with Engineering Applications, McGrawHill, New York, NY, USA, 1997.

[12] E. H. Mamdani and S. Assilian, "An experiment in linguistic synthesis with a fuzzy logic controller," International Journal of Man-Machine Studies, vol. 7, no. 1, pp. 1-13, 1975.

[13] Y. Xueshan, Q. Xiaozhai, G. C. Lee, M. Tong, and C. Jinming, "Jerk and jerk sensor," in Proceedings of the 14th World Conference on Earthquake Engineering, Beijing, China, October 2008.

[14] T. Sato et al., Enhancement of Earthquake Performance of Infrastructures Based on Investigation into Fracturing Process, EEPI, Kyoto University, March 2002.

[15] F. Schiefer and G.-P. Ostermeyer, "A contribution to jerk detection," Proceedings in Applied Mathematics and Mechanics, vol. 9, no. 1, pp. 125-126, 2009. 


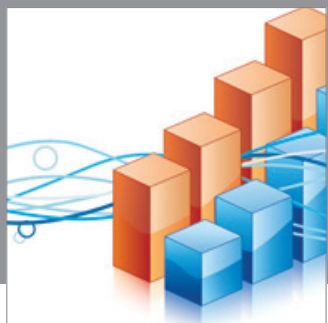

Advances in

Operations Research

mansans

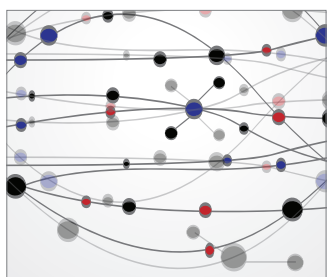

The Scientific World Journal
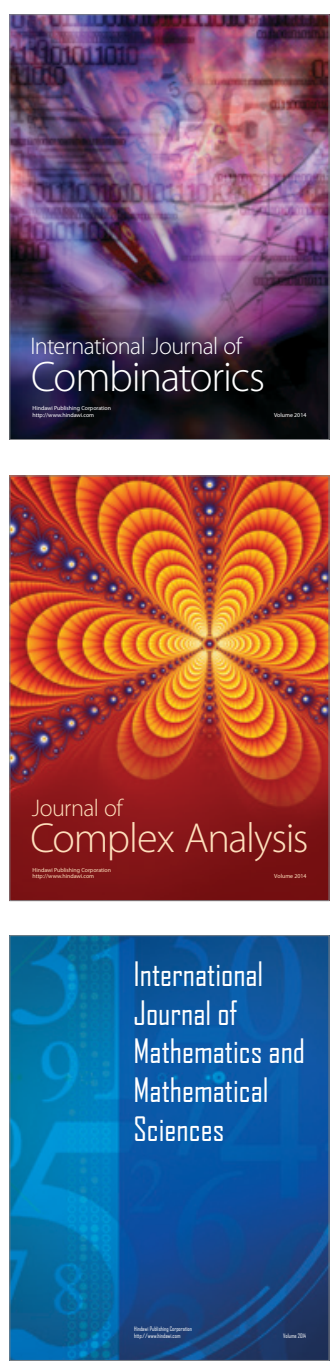
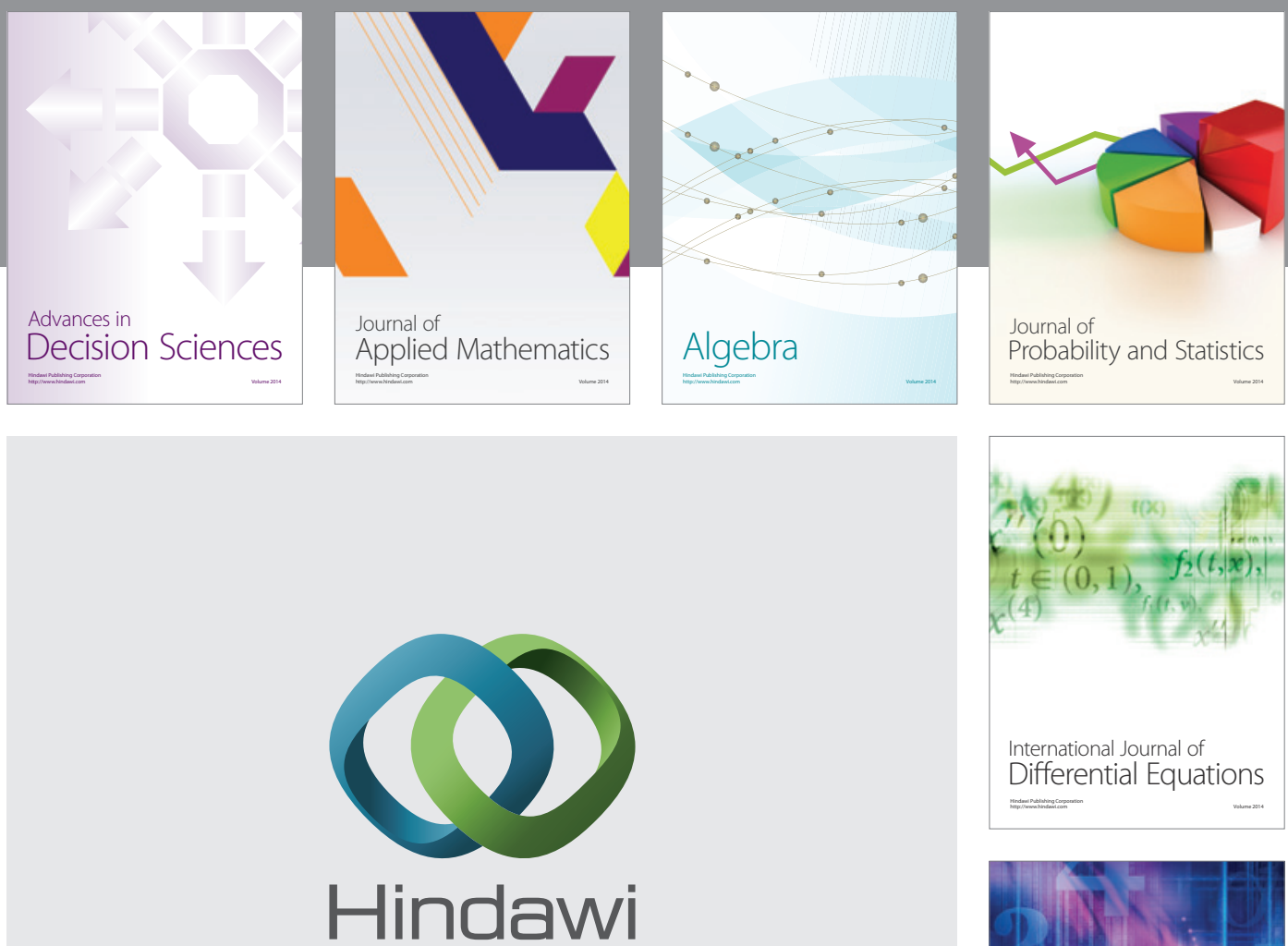

Submit your manuscripts at http://www.hindawi.com
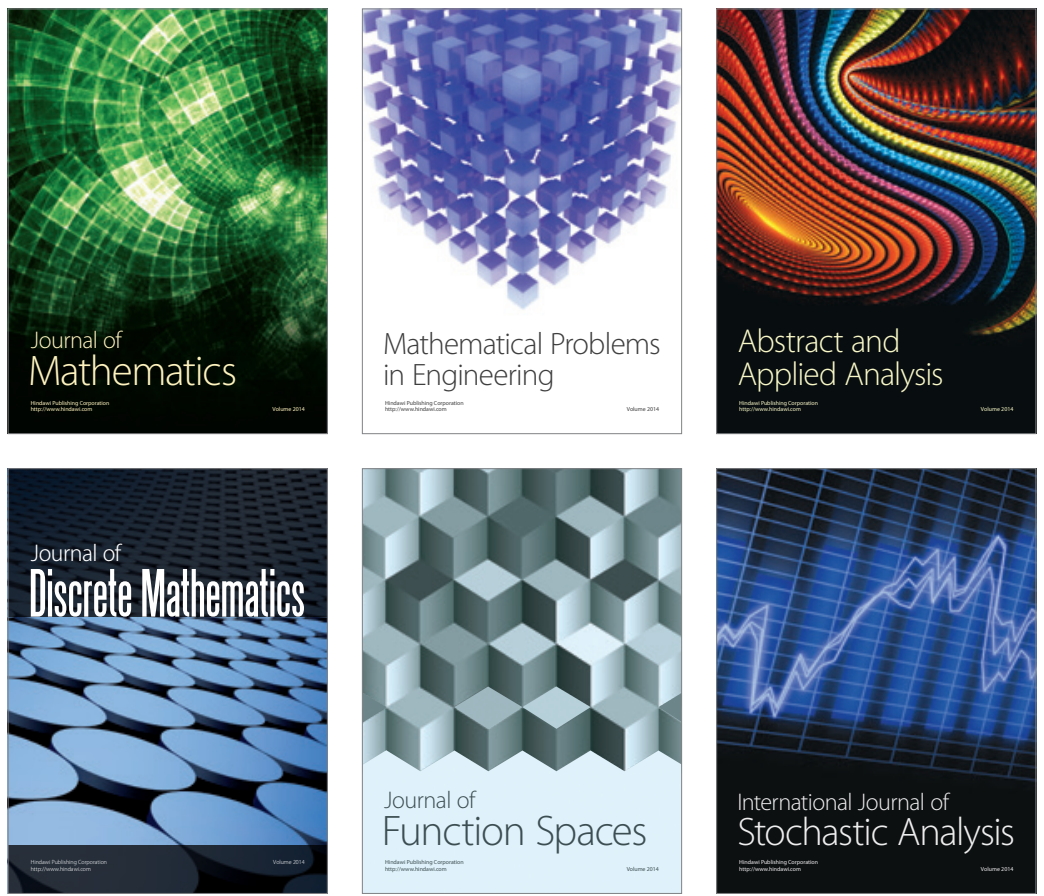

Journal of

Function Spaces

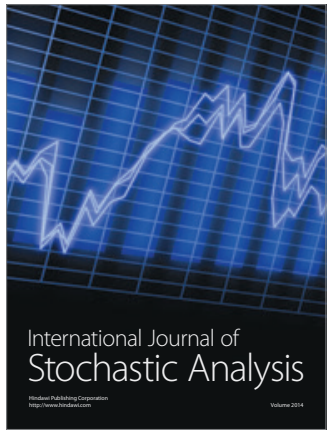

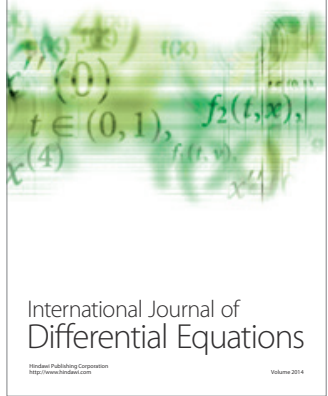
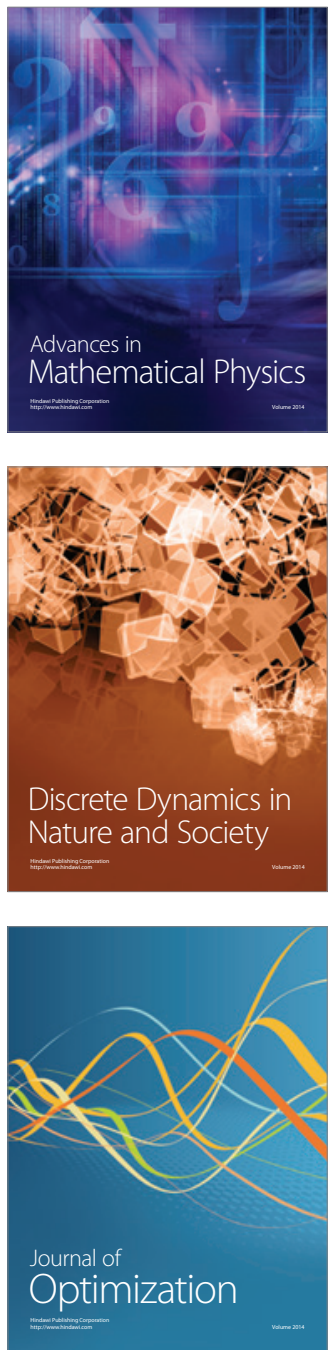